\title{
Structural Insights into the Substrate Promiscuity of a Laboratory-Evolved Peroxygenase
}

\author{
Mercedes Ramirez-Escudero, ${ }^{\dagger, \|}$ Patricia Molina-Espeja, ${ }^{\ddagger}\|\|$ Patricia Gomez de Santos, \\ Martin Hofrichter, ${ }^{\S}$ Julia Sanz-Aparicio, ${ }^{* \dagger}{ }^{\dagger}$ and Miguel Alcalde ${ }^{*},+0$ \\ ${ }^{\dagger}$ Department of Crystallography \& Structural Biology, Institute of Physical Chemistry "Rocasolano”, CSIC, 28006 Madrid, Spain \\ "Department of Biocatalysis, Institute of Catalysis, CSIC, 28049 Madrid, Spain \\ ${ }^{\S}$ Department of Bio- and Environmental Sciences, TU Dresden, International Institute Zittau, Mark 23, 02763 Zittau, Germany
}

\author{
Supporting Information
}

ABSTRACT: Because of their minimal requirements, substrate promiscuity and product selectivity, fungal peroxygenases are now considered to be the jewel in the crown of $\mathrm{C}-\mathrm{H}$ oxyfunctionalization biocatalysts. In this work, the crystal structure of the first laboratory-evolved peroxygenase expressed by yeast was determined at a resolution of $1.5 \AA$. Notable differences were detected between the evolved and native peroxygenase from Agrocybe aegerita, including the presence of a full $\mathrm{N}$-terminus and a broader heme access channel due to the mutations that accumulated through directed evolution. Further mutagenesis and soaking experiments with a palette of peroxygenative and peroxidative substrates suggested dynamic trafficking through the heme channel as the main driving force for the exceptional substrate promiscuity of peroxygenase. Accordingly, this study provides the first structural evidence at an atomic level regarding the mode of substrate binding for this versatile biocatalyst, which is discussed within a biological and chemical context.

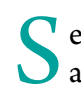
elective oxyfunctionalizations of organic molecules are among the most attractive transformations in synthetic chemistry. ${ }^{1}$ Given that the use of transition metal catalysts to activate inert $\mathrm{C}-\mathrm{H}$ bonds is strongly associated with poor selectivity and harsh conditions, ${ }^{2}$ hope of performing such reactions under milder conditions remains, encouraged by the fact that such processes are carried out, as a matter of routine, within biological networks. Indeed, for more than 30 years, cytochrome P450 monooxygenases have been considered as promising biocatalysts for achieving such a milestone in contemporary chemistry. ${ }^{3,4}$ However, and despite their exquisite regio-, chemo-, and enantioselectivity, the use of isolated P450s on an industrial scale is littered with hurdles, which range from their dependence on expensive redox cofactors (e.g., NADPH) and auxiliary flavoproteins to a lack of stability in the face of the demanding industrial conditions and their common association with membranes. More significantly, P450s suffer from uncoupling reactions (i.e., the reducing power is diverted from the target reaction to futile reduction reactions, the so-called oxygen dilemma that is linked to their inherent catalytic mechanism). ${ }^{5-8}$ Different steps have been taken to overcome these limitations, engineering more efficient and self-sufficient P450s, for example, working through the peroxide shunt pathway by using $\mathrm{H}_{2} \mathrm{O}_{2}$ as the main oxygen source and the final electron acceptor, just like an "artificial" peroxygenase," 3,9 or more recently to lead their promiscuity toward non-natural chemistry. ${ }^{10}$ In addition, several studies have used natural P450 peroxygenases (i.e., fueled by $\mathrm{H}_{2} \mathrm{O}_{2}$ ) that catalyze the $\alpha$-selective hydroxylation of long fatty acids. ${ }^{11-13}$ Meanwhile, the first extracellular natural peroxygenase was identified and isolated from the fungal kingdom in 2004, specifically from the basidiomycete Agrocybe aegerita, ${ }^{14}$ and it was characterized at the genome level in $2009 .^{15}$ Less than one decade later, more than 4000 peroxygenase-like gene sequences have been deposited in genomic databases, and fungal peroxygenases are now considered by many scientists as the ideal biocatalyst for oxyfunctionalization chemistry. ${ }^{16-18}$ Because of its "chimeric" properties (bringing together the catalytic attributes of $\mathrm{P} 450 \mathrm{~s}$, classical heme-peroxidases, and chloroperoxidase from Caldariomyces fumago, CPO), it has been termed unspecific peroxygenase (UPO) and recognized as the first member of a new sub-subclass of oxidoreductases (EC 1.11.2.1). Through a hybrid catalytic mechanism, UPO shows both peroxidative activity (one-electron oxidation, e.g., of phenolics) and, more notably, peroxygenative activity (oxygen-transferring two-electron oxidation with peroxide as the oxygen source) (Supplementary Scheme 1). The latter drives the promiscuity of UPOs in a wide portfolio of transformations that includes aromatic, alkylic, (cyclo)aliphatic, and heterocyclic hydroxylations,

Received: May 29, 2018

Accepted: October 30, 2018

Published: October 30, 2018 


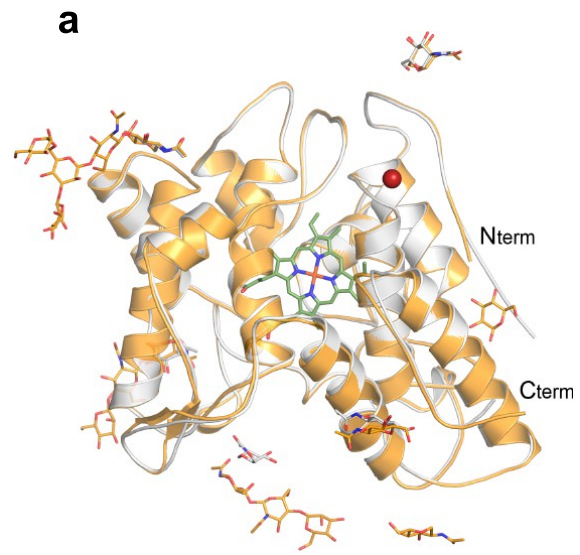

b

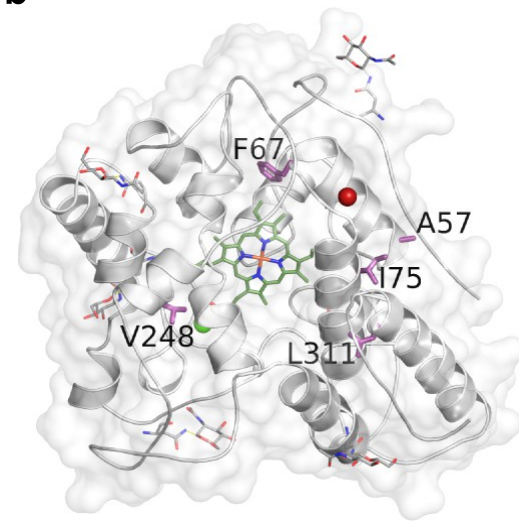

Figure 1. General view of the evolved peroxygenase structure at $1.5 \AA$ resolution with a ligand-free active site. (a) The cartoon represents the overall structure of native (light orange) vs PaDa-I (white), showing the magnesium ion $\left(\mathrm{Mg}^{2+}\right.$, green sphere), the heme group (dark green sticks), the chloride ion (red sphere), and the different glycan chains as sticks. (b) The residues mutated in the evolved peroxygenase are highlighted as purple sticks. Protein Data Bank entry 5OXU (see also Table 1).

aromatic and aliphatic olefin epoxidations, ether cleavages (O-dealkylations), $\mathrm{N}$-dealkylations, sulfoxidations, $\mathrm{N}$-oxidations, deacylations ( $\mathrm{C}-\mathrm{C}$ bond cleavages), and halide oxidations/ halogenations. ${ }^{18-21}$

To adjust the catalytic properties of this enzyme and satisfy the needs of industry, heterologous production of UPOs is essential. As such, we achieved the functional expression (secretion) of an UPO from A. aegerita (AaeUPO) in Saccharomyces cerevisiae and Pichia pastoris through several rounds of laboratory evolution. ${ }^{8,22,23}$ With expression levels of $8 \mathrm{mg} / \mathrm{L}$ in S. cerevisiae and $>200 \mathrm{mg} / \mathrm{L}$ in $P$. pastoris in a bioreactor, the highly active and stable AaeUPO secretion variant (called PaDa-I) was very recently subjected to new directed evolution enterprises aimed at producing, with high efficiency, agrochemicals and relevant pharma compounds. ${ }^{24,25}$ Within the same framework, the crystal structure of native AaeUPO was published at a resolution of $\sim 2.2 \AA^{26}$ It is a compact monomeric protein comprised of $10 \alpha$-helices and five short $\beta$-sheets with a catalytic pocket carved into the center of the structure through a cone-shaped channel (frustum) that is formed by hydrophobic-aromatic amino acids. Buried at its apex, this channel has a protoporphyrin IX that carries a ferric iron, constituting the heme prosthetic group. AaeUPO also has a cysteine residue (Cys36) as the axial (fifth) ligand of the heme, which situates UPOs along with CPO and P450s in the category of the hemethiolate enzymes. ${ }^{17}$

Understanding the mode of substrate binding in UPO will help answer key questions regarding the biological and chemical activity of this versatile biocatalyst. In turn, this information will open the way to design "smarter" mutant libraries, combining computational and experimental tools in a drive toward the generation of more efficient, industrially competent peroxygenases. ${ }^{27}$ Here we present a thorough study of the mode of substrate binding used by UPO with a complete set of peroxidative and peroxygenative substrates. First, the evolved $\mathrm{PaDa}-\mathrm{I}$ mutant was crystallized, and its high-resolution structure was compared to that of native AaeUPO. Significant differences between the two templates were found, which were rationalized in further mutagenesis experiments. Subsequently, a broad palette of peroxidative and peroxygenative compounds were used to form complexes with the $\mathrm{PaDa}$-I mutant to obtain the first experimental evidence of the patterns of substrate binding for this multipurpose biocatalyst.

\section{RESULTS}

Structural Analysis of the Evolved Peroxygenase. Crystallization of the Ligand-Free Mutant and Its General Structure. The PaDa-I mutant is the outcome of five rounds of directed evolution to enhance its activity and its heterologous functional expression in $S$. cerevisiae. ${ }^{22}$ Carrying nine mutations (F12Y-A14V-R15G-A21D-V57A-L67F-V75I-I248V-F311L, the underlined mutations lying in the signal peptide), this evolved recombinant peroxygenase is also readily secreted by $P$. pastoris under the control of the AOX1 promoter in a highly stable and active form and at aood titer while preserving the properties obtained after laboratory evolution in S. cerevisiae. ${ }^{23}$ $\mathrm{PaDa}-\mathrm{I}$ has a strong degree of glycosylation [30\%, molecular weight (MW) of $51.1 \mathrm{kDa}$ ], which makes it difficult to obtain high-quality crystals. To reduce the heterogeneity of the sample, the mutant produced by $P$. pastoris in a bioreactor and purified to homogeneity (Reinheitszahl value, $R z_{A_{418} / A_{280}} \sim 2$ ) was treated with Endo H, and its MW decreased to $37 \mathrm{kDa}$ (Supplementary Figure 1a,b). Preliminary crystals of deglycosylated peroxygenase appeared with 20\% PEG 3350 and $0.2 \mathrm{M}$ $\mathrm{NH}_{4} \mathrm{NO}_{3}$ ( $\mathrm{pH}$ 6.3), and they were further optimized by seeding and buffering the solution in $0.1 \mathrm{M}$ sodium acetate ( $\mathrm{pH}$ 4.0). These crystals diffracted to a maximum resolution of $1.4 \AA$ but had an acetate anion bound to the active site, which also occurred in the crystal of native AaeUPO in sodium acetate buffer. ${ }^{26}$ Because acetate is a peroxygenase inhibitor, subsequent crystallization trials were performed with cacodylate, citrate, and phosphate buffers, although nitrate was then found associated with the active site. Finally, high-quality peroxygenase crystals without any molecule attached to the active site were obtained in $1.5-1.8 \mathrm{M} \mathrm{Na} / \mathrm{K}$ phosphate buffer ( $\mathrm{pH}$ 5.6) with 3-10\% 2-methyl-2,4-pentanediol (MPD). Under such conditions, crystals of the enzyme with a ligandfree active site were obtained at the highest resolution reported so far for a fungal peroxygenase, $1.5 \AA$ (a full description of the experimental and structural measurements is provided in Table 1 and in the Materials and Methods of the Supporting Information).

The crystals belonged to the $P 2_{1}$ space group, with one molecule per asymmetric unit. Endo $\mathrm{H}$ cleaves oligosaccharide moieties to leave single $N$-acetylglucosamine (GlcNAc) units, although poorly accessible glycosylation sites may have remained partially glycosylated. Recombinant PaDa-I expressed 
裹新

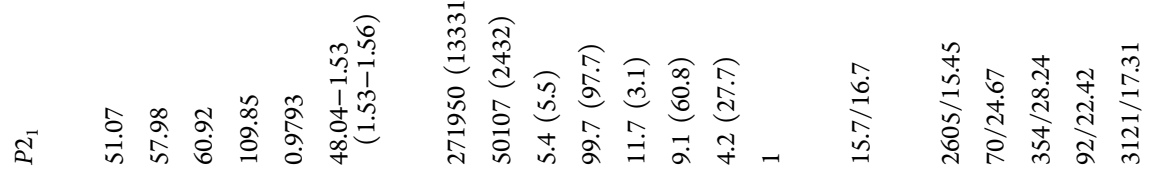

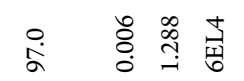

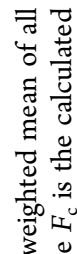

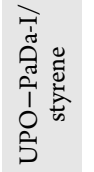

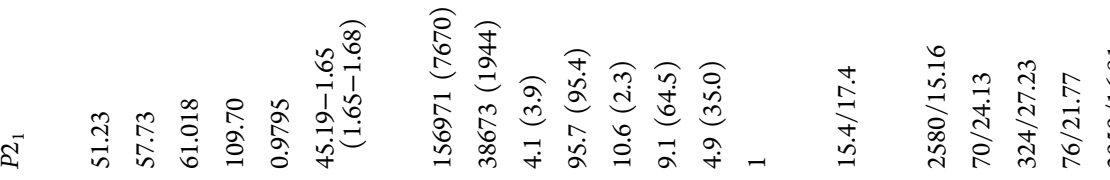

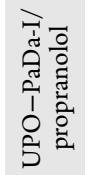

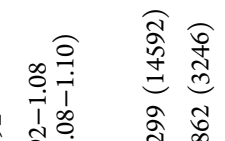

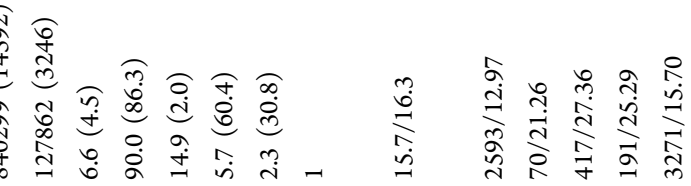

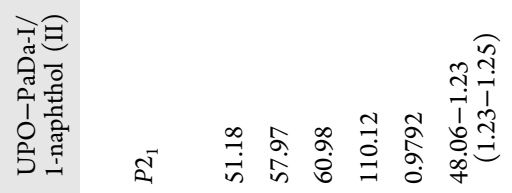

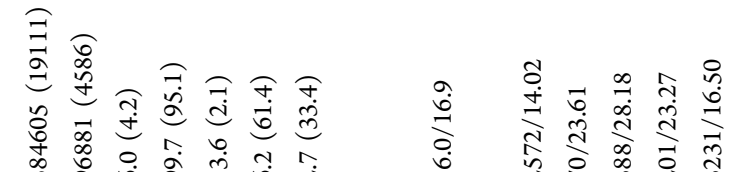

:

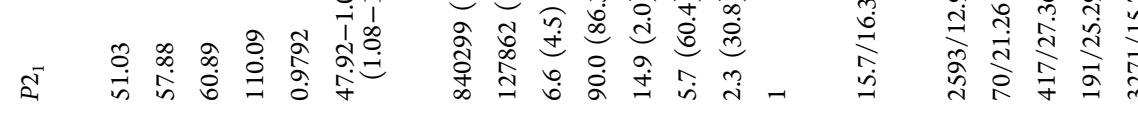

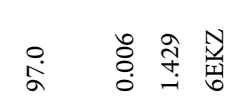

핀

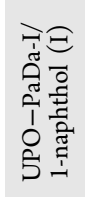

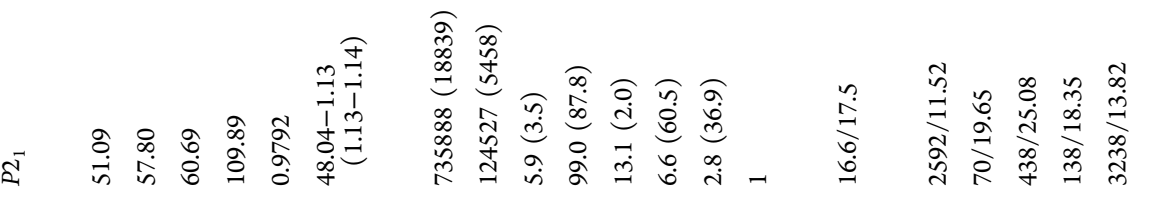

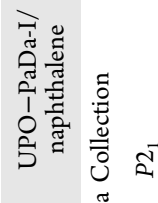

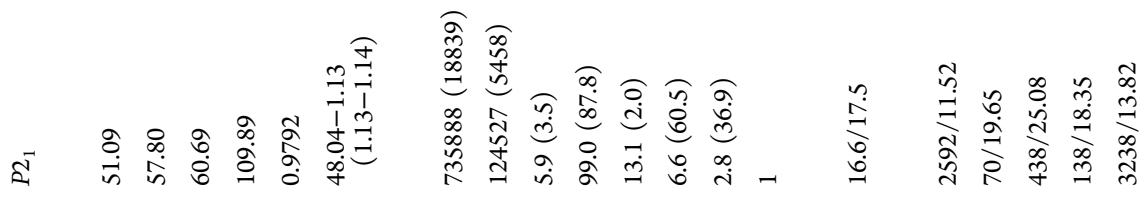

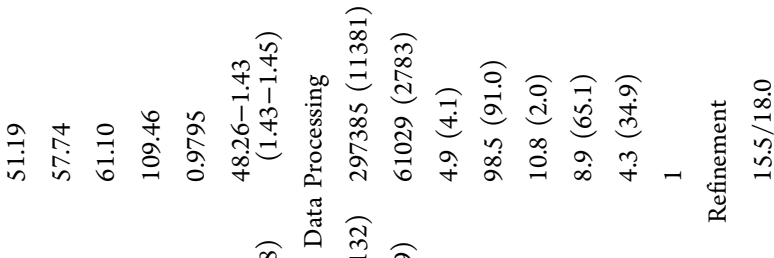

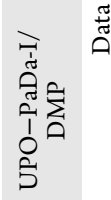

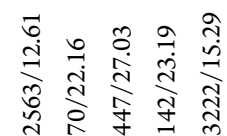

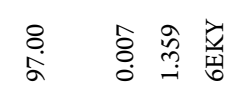

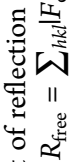

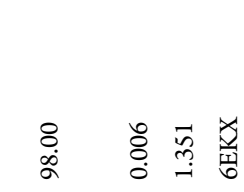

离通

도

㝳

$\stackrel{2}{1}$ 
in P. pastoris is more strongly glycosylated $(30 \%)^{23}$ than native AaeUPO (22\%), with six potential glycosylation sites (as determined for the wild type enzyme). The electron density maps allowed modeling of the GlcNAc units, and they were attached to Asn11, Asn141, Asn161, Asn182, Asn286, and Asn295. We measured the kinetic parameters of PaDa-I from $P$. pastoris [before and after deglycosylation (Supplementary Figure 1c)] and found that the deglycosylated enzyme conserved its activity on both peroxidative and peroxygenative substrates.

The final structure of $\mathrm{PaDa}-\mathrm{I}$ with a ligand-free active site is formed by the polypeptide chain Glu1-Arg327 (harboring the mutational backbone V57A-L67F-V75I-I248V-F311L), and it has an overall structural arrangement similar to that described for native AaeUPO (Figure 1). PaDa-I is composed of 10 $\alpha$-helices linked by long loops. According to SCOP2, ${ }^{28} \mathrm{PaDa}-\mathrm{I}$ belongs to the all $\alpha$ class of common heme-containing proteins, with the heme cofactor deeply embedded in the helices and surrounded by loops. The evolved peroxygenase includes the protoporphyrin IX (heme group) with the iron hexacoordinated, with its fifth position associated with the characteristic proximal Cys36 (axial) ligand and the sixth position (distal ligand) with a water molecule. The Glu196-Arg189 pair forms the distinctive acid-base pair required for binding and heterolytic cleavage of peroxide, which leads to enzyme activation via consecutive formation of the catalytic intermediates Compound 0 (ferric-peroxo complex) and Compound I (ferryl-oxo complex). Compound I is the reactive key intermediate that can follow two different pathways depending on the particular substrate: (i) "two-electron oxidation" of one substrate molecule along with oxygen atom transfer (peroxygenative activity) or (ii) two "one-electron oxidations" of two substrate molecules resulting in the formation of the corresponding substrate radicals (e.g., phenoxyl radicals; peroxidative activity) ${ }^{17}$ (Supplementary Scheme 1). The heme access channel is upholstered by 9 aromatic residues (eight Phe residues and one Tyr residue), of which Phe69, Phe121, and Phe199 form a triad that orients the substrate toward the heme, whereas Phe76 and Phe191 delimit the entrance of the channel. ${ }^{26}$ As in native AaeUPO, the heme channel is a $17 \AA$ long deep funnel (conical frustum). PaDa-I has a structural magnesium $\left(\mathrm{Mg}^{2+}\right.$, green sphere, in Figure 1), which may stabilize the porphyrin ring system, with the same coordination as that reported previously for native AaeUPO. ${ }^{26}$ Also, similar to wild type AaeUPO and chloroperoxidase $\left(\mathrm{CPO}^{29}\right)$, PaDa-I possesses at least one halide binding site near the enzyme's channel entrance where, for example, chloride can bind (Figure 1b, red sphere). A phosphate anion can also be found within a positively charged region of the PaDa-I crystals, like the sulfate found in crystals of the wild type enzyme. Furthermore, several glycerol molecules from the cryoprotectant solution were bound to different positions of the molecular surface.

Structural Differences between the Evolved and Native Peroxygenases. As in native AaeUPO, the C-terminus of the evolved $\mathrm{PaDa}-\mathrm{I}$ is stabilized by the Cys278-Cys319 disulfide bridge while the $\mathrm{N}$-terminus is markedly different. The PaDa-I crystal maintains the complete $\mathrm{N}$-terminal region, which, in the wild type enzyme, undergoes proteolysis at Gly3-Leu4 (EPG/ LPP) $)^{26}$ (Figure 1a,b). This observation was confirmed by $\mathrm{N}$-terminal sequencing of PaDa-I expressed by $\mathrm{S}$. cerevisiae and $P$. pastoris, each with an identical N-terminus (EPGLPPPGPL). The lack of proteolysis at the N-terminus of recombinant
PaDa-I may be due to the introduction of the V57A and V75I mutations by directed evolution, although we cannot rule out the absence of efficient proteases in the heterologous hosts. These substitutions are located in the inner part of two helices that flank the N-terminal Pro5-Pro6 segment, and they produce a shift of $1 \AA$ at Pro5 that may modify the existing hydrophobic contacts, possibly impeding the $\mathrm{N}$-terminal processing.

Among other mutations added by directed evolution, the L67F mutation is buried in a hydrophobic pocket defined by Phe204, Phe222, and Phe232, and it is located in the vicinity of the heme group, making hydrophobic contact with one of its methylene groups. While Phe67 retains the same pattern of interactions with the surrounding aromatic residues, producing no evidence of structural changes, we cannot rule out electronic effects in the protoporphyrin ring caused by the substitution. Finally, the I248V and F311L mutations are placed in positions with the side chains oriented toward the heme channel, shaping the active site for ligand binding. Ile248 is in a buried hydrophobic pocket far from the heme, and its replacement with Val did not result in structural modifications. By contrast, the F311L mutation produced the most significant changes in the structure of $\mathrm{PaDa}-\mathrm{I}$, along with the aforementioned complete $\mathrm{N}$-terminus (Figure $2 \mathrm{a}-\mathrm{d}$ ).

Phe311 lies $3.6 \AA$ from Phe76, establishing a hydrophobic contact (as shown in Figure 2b). Both Phe76 and Phe191 form a pair of protruding aromatic residues at the entrance of the heme access channel that are supposed to guide the substrate to the active site, also establishing hydrophobic contacts in native AaeUPO. The change of a bulky Phe to a smaller Leu at position 311 shifts the Phe76 side chain but preserves the interaction with Leu311 while weakening the contact with Phe191 (Figure 2c). This change possibly leaves Phe191 more exposed to the solvent, with a dual conformation observed for this residue (Figure 2a), as also evident in the soaking experiments (see below). Indeed, the modification provoked a marked switch in the position of the Phe191 side chain that reorients to establish a stabilizing interaction with Phe274 at a distance of $3.7 \AA$ (Figure 2c). The consequence is a larger entrance to the heme channel, with distances between Phe191 and Phe76 of 4.1 and $7.8 \AA$ in native AaeUPO and PaDa-I, respectively (Figure 2a,d).

To assess the role played by F311L, we reverted this mutation by site-directed mutagenesis and characterized the purified reverted $\mathrm{PaDa}-\mathrm{I}-\mathrm{rev}$ mutant biochemically. The kinetic thermostability of this mutant was not compromised, with the same $T_{50}$ value as PaDa-I $\left(58{ }^{\circ} \mathrm{C}\right)$. When the apparent steady-state kinetic parameters for its peroxidative [2,2' -azino-bis(3-ethylbenzothiazoline-6-sulfonic acid) (ABTS) and 2,6-dimethoxyphenol (DMP)] and peroxygenative [veratryl alcohol (VA) and 5-nitro-1,3-benzodioxole (NBD)] substrates were measured, PaDa-I-rev showed 4- and 3-fold enhancements in the $k_{\text {cat }} / K_{\mathrm{m}}$ for ABTS and VA, respectively, whereas the catalytic efficiencies for DMP and NBD were practically conserved (Table 2). Strikingly, the secretion level was reduced by roughly $70 \%$ relative to that of $\mathrm{PaDa}-\mathrm{I}$, such that the $\mathrm{F} 311 \mathrm{~L}$ mutation necessarily exerts a beneficial effect on folding and expression, while its influence on activity and selectivity may vary depending on the substrate and the reaction catalyzed.

Given the observed plasticity of the heme access channel, with a dual conformational state for Phe191 and a broader entrance after the F311L mutation, we explored other positions involved in such effects. The Ala316 at the entrance of the funnel (Figure 2d) is located in a flexible loop (G314-G318) 

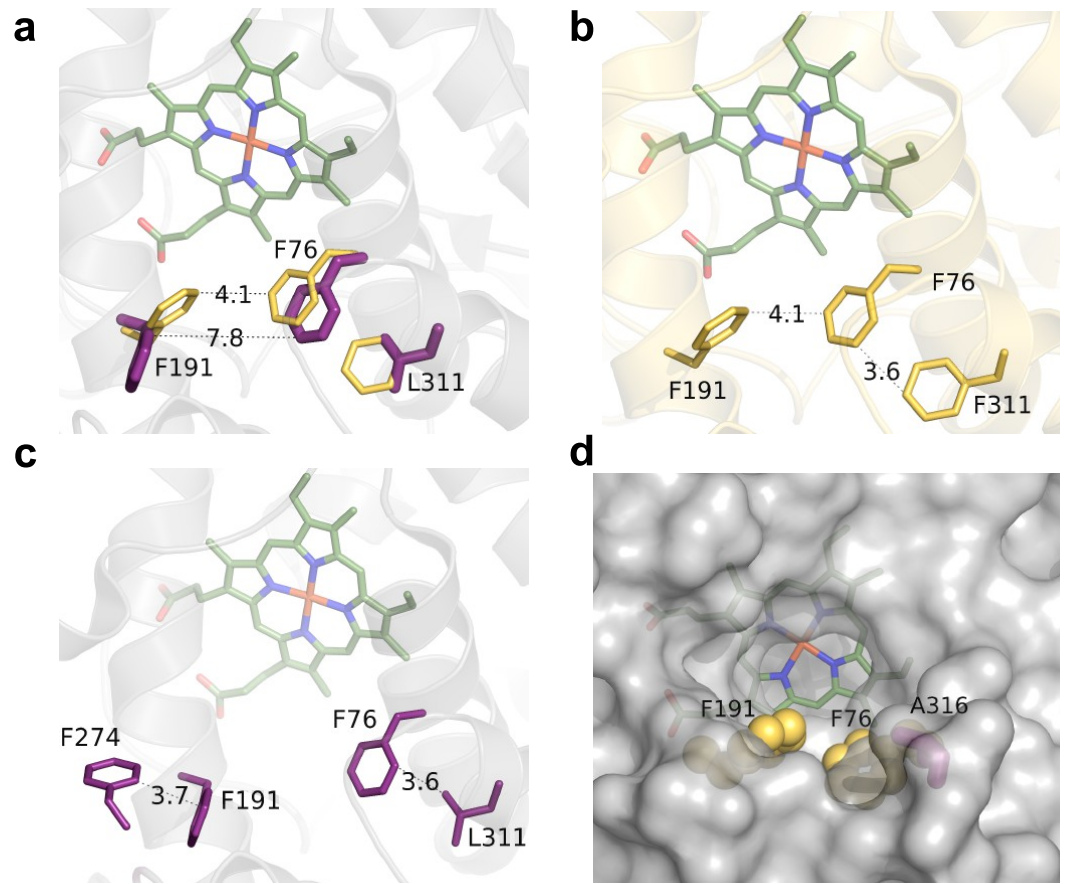

Figure 2. Active site differences between evolved and native peroxygenase. (a) The F311L change (wild type AaeUPO, yellow; PaDa-I, purple) produces a shift in the position of Phe76 that affects the Phe191 side chain, enlarging the entrance to the heme (green) channel. Details of the atomic interactions between relevant residues in (b) wild type AaeUPO or (c) recombinant PaDa-I crystal structures. (d) The surface representation of PaDa-I illustrates the funnel that provides access to the heme group. The equivalent positions of Phe76 and Phe191 in the wild type AaeUPO structure (yellow spheres) show the expanded entrance in PaDa-I. Ala316 is highlighted as a violet stick. PaDa-I, Protein Data Bank (PDB) entry 5OXU; AaeUPO, PDB entry 2YOR (see also Table 1).

that also shows distinct conformational states upon substrate binding (see below), and this seems to compromise the accessibility to the heme. Accordingly, this residue was studied by saturation mutagenesis, and screening of the corresponding mutant library with ABTS and NBD unveiled the JEd-I variant (carrying the A316P mutation). The kinetics of purified JEd-I were enhanced for all the substrates tested, with an improvement in the $k_{\mathrm{cat}} / K_{\mathrm{m}}$ that ranged from 1.5 - to 6 -fold, while its thermostability was conserved (Table 2). The change of Ala to Pro would be expected to decrease the flexibility of this loop, although rigidity is not usually related to improved activity. While the precise effect of this substitution may be difficult to explain, these results do address the ease with which the malleability of the heme access channel can be manipulated and its direct implication on the broad UPO substrate specificity.

Substrate Binding Mode. PaDa-I mutant crystals were examined in soaking experiments with panels of peroxidative (ABTS, DMP, 1-naphthol, and 2-naphthol) and peroxygenative substrates [naphthalene, propranolol, diclofenac, acetanilide, styrene, NBD, VA, benzyl alcohol (BA), and palmitic acid]. Productive crystal complexes were obtained with many of the substrates (DMP, 1-naphthol, naphthalene, propranolol, styrene, and VA) at a catalytic distance from the heme of $\sim 4 \AA$ (Supplementary Figure 2). Full details about the crystallography of PaDa-I complexes can be found in the Materials and Methods of the Supporting Information and in Table 1. The other substrates were associated with low occupancy or a disordered active site, and they were not included in the analysis. As mentioned above, the F311L modification in the PaDa-I mutant enhanced the accessibility of the substrates to the heme catalytic site by increasing the size of the outer entrance to the channel from 8 to $12 \AA$ (Figure $2 \mathrm{a}, \mathrm{d}$ ). The switch in the
Phe191 side chain found in PaDa-I was also evident in all the complexes obtained, in some cases with the dual conformation observed in the ligand-free PaDa-I crystal (Figures 2a and 3a,e).

All the complexes obtained, including those with substrates for the peroxidase reaction, showed a clear electron density within the heme channel that situates ligand binding in van der Waals contact with the heme group, indicating that catalysis is mostly performed by direct interaction with the $\mathrm{Fe}$ protoporphyrin IX cofactor (Figure $3 \mathrm{a}-\mathrm{i}$ ). Moreover, a water molecule is bound between the heme and the substrate, in a position similar to that reported for the distal oxygen of the ferric hydroperoxo intermediate, denoted as Compound 0 and representing the first step in the reaction pathway. ${ }^{30}$ Consequently, the complexes provided features relevant to substrate recognition that are necessary for the reaction to occur. Although the residues defining the channel are mostly hydrophobic, the funnel is occupied by an extended net of water molecules that is ordered through hydrogen bonds with the main chain of different residues, thereby shaping the channel. In the soaking experiments, the ligands displaced some of these water molecules upon binding. The determined structure of the complexes revealed that all the substrates sandwich their aromatic ring between Phe121 and Phe199, mostly in a T-interaction mode, also contacting the hydrophobic Phe69.

Panels $\mathrm{a}$ and $\mathrm{b}$ of Figure 3 show the bound acetate ion and DMSO, respectively, two strong peroxygenase inhibitors, trapped from the medium and blocking the access of the substrates to the active site. This phenomenon was reported previously for acetate bound to wild type AaeUPO ${ }^{26}$ and also for $\mathrm{CPO}^{30}$ and the dimeric Marasmius rotula peroxygenase [MroUPO, Protein Data Bank (PDB) entry 5FUJ, unpublished, K. Piontek, E. Strittmatter, and D. A. Plattner, Institute of Organic 
Table 2. Apparent Kinetic Parameters of Evolved Peroxygenases ${ }^{a}$

\begin{tabular}{|c|c|c|c|c|}
\hline substrate & kinetic constant & PaDa-I & PaDa-I-rev & JEd-I \\
\hline \multirow[t]{3}{*}{ ABTS } & $K_{\mathrm{m}}(\mathrm{mM})$ & $0.12 \pm 0.01$ & $0.046 \pm 0.008$ & $0.027 \pm 0.006$ \\
\hline & $k_{\mathrm{cat}}\left(\mathrm{s}^{-1}\right)$ & $965 \pm 23$ & $1359 \pm 31.5$ & $1220 \pm 25$ \\
\hline & $k_{\mathrm{cat}} / K_{\mathrm{m}}\left(\mathrm{mM}^{-1} \mathrm{~s}^{-1}\right)$ & $7457 \pm 276$ & $29856 \pm 4368$ & $45031 \pm 8828$ \\
\hline \multirow[t]{3}{*}{ DMP } & $K_{\mathrm{m}}(\mathrm{mM})$ & $0.38 \pm 0.02$ & $0.31 \pm 0.002$ & $0.23 \pm 0.03$ \\
\hline & $k_{\text {cat }}\left(\mathrm{s}^{-1}\right)$ & $175 \pm 5$ & $165 \pm 4.6$ & $166 \pm 6$ \\
\hline & $k_{\mathrm{cat}} / K_{\mathrm{m}}\left(\mathrm{mM}^{-1} \mathrm{~s}^{-1}\right)$ & $464 \pm 17$ & $528 \pm 23$ & $706 \pm 52$ \\
\hline \multirow[t]{3}{*}{ VA } & $K_{\mathrm{m}}(\mathrm{mM})$ & $10.3 \pm 1.4$ & $6.6 \pm 0.7$ & $5.8 \pm 0.5$ \\
\hline & $k_{\text {cat }}\left(\mathrm{s}^{-1}\right)$ & $75 \pm 5$ & $152 \pm 6$ & $191 \pm 5$ \\
\hline & $k_{\mathrm{cat}} / K_{\mathrm{m}}\left(\mathrm{mM}^{-1} \mathrm{~s}^{-1}\right)$ & $7.3 \pm 0.6$ & $23 \pm 1.7$ & $33 \pm 2$ \\
\hline \multirow[t]{3}{*}{ NBD } & $K_{\mathrm{m}}(\mathrm{mM})$ & $1 \pm 0.11$ & $0.71 \pm 0.1$ & $0.91 \pm 0.08$ \\
\hline & $k_{\text {cat }}\left(s^{-1}\right)$ & $412 \pm 21$ & $307 \pm 18$ & $491 \pm 21.2$ \\
\hline & $k_{\text {cat }} / K_{\mathrm{m}}\left(\mathrm{mM}^{-1} \mathrm{~s}^{-1}\right)$ & $389 \pm 22$ & $430 \pm 37$ & $541 \pm 28$ \\
\hline
\end{tabular}

${ }^{a}$ Kinetic constants of UPO variants for ABTS were determined in $100 \mathrm{mM}$ sodium citrate/phosphate buffer $(\mathrm{pH} 4.0)$ containing 2 mM $\mathrm{H}_{2} \mathrm{O}_{2}$; the other substrates were tested in $100 \mathrm{mM}$ potassium phosphate ( $\mathrm{pH} 7.0)$ containing $1 \mathrm{mM} \mathrm{H}_{2} \mathrm{O}_{2}\left(\mathrm{NBD}, 15 \%\right.$ acetonitrile) or $2 \mathrm{mM} \mathrm{H} \mathrm{H}_{2}(\mathrm{DMP}$ and VA). Variants were produced in S. cerevisiae and purified to homogeneity with $R z$ values of 2.2 (PaDa-I), 1.6 (PaDa-I-rev), and 1.7 (JEd-I).

Chemistry, University of Freiburg, Freiburg, Germany]. Moreover, a glycerol molecule trapped from the cryoprotectant solution showed a similar mode of binding in the crystals soaked into the $\beta$ blocker propranolol (Figure 3c). The substrate was found at two positions within the channel, both far from the heme site.

In the deposited structure of MroUPO in a complex with propranolol (PDB entry 5FUK, unpublished, K. Piontek, E. Strittmatter, and D. A. Plattner, Institute of Organic Chemistry, University of Freiburg, Freiburg, Germany), the ligand is also bound at a position far from the heme. However, in that case, the substrate competes with a partially occupied palmitic acid molecule that spans the catalytic site. Nevertheless, our crystals reveal that UPO can allocate the substrate propranolol to ordered positions within the channel, emulating the entrance/ exit of the pathway toward the heme catalytic site. In this respect, it is worth noting that we recently reported the in vitro evolution of UPO for the selective hydroxylation of propranolol and, by computational analysis, defined the gradual diffusion of propranolol through the heme channel as the main factor for a correct binding at a distance of $\sim 4 \AA$ from the catalytic heme-ferryl oxygen. $^{25}$

The peroxygenative substrate naphthalene and its hydroxylation product 1-naphthol (a peroxidative substrate itself) also bound at a position situated $4 \AA$ from the heme, with a water molecule in the middle that mimics the heme oxygen as indicated above (Figure $3 \mathrm{~d}-\mathrm{f}$ ). The bicyclic aromatic ring is sandwiched between Phe121 and Phe199 in a T-packing mode, with an additional strong hydrophobic interaction with Phe69 that maintains a highly conserved orientation with respect to the heme plane in all the crystals analyzed. However, some disorder is observed in the electron density of many crystals at the second fused ring and in the positions of the hydroxyl groups, possibly indicating dynamic nonspecific binding. In this regard, when we consider two independent complexes obtained after soaking in 1-naphthol for different periods of time, one of them contains two molecules of the substrate passing along the heme channel with a well-defined density (Figure 3e, 1-naphthol complex I). In the other crystal, there were five molecules of the substrate occupying the channel (Figure 3f, 1-naphthol, complex II), although the position of the hydroxyl group could not be unambiguously assigned in the molecule next to the heme, probably because of its dynamic binding. A similar binding pattern was observed in DMP-soaked crystals (Figure 3g), which had the phenolic ring oriented similarly by the three Phe residues. It should be noted that the complex of the dyedecolorizing peroxidase $(\mathrm{DyP})$ with this peroxidative substrate reported previously had the DMP molecule bound at a position far from the heme site, in which electron transfer occurs through a net of water molecules connecting the substrate to the heme ion. ${ }^{31}$ By contrast, in our complex, the aromatic ring was clearly located $4 \AA$ from the heme and included the ferric water, which may indicate the alternative nature of DMP both as a peroxidative substrate and as a peroxygenative substrate. Indeed, it is reasonable to think that, besides phenoxyl radical formation, the "aromatic double bond" between C4 and C5 in DMP is epoxidized, leading, after spontaneous rearrangement and rearomatization, to 2,6-dimethoxyhydroquinone (Figure 3g). Moreover, it should be noted that two conformations of the catalytic Glu196 of the acid-base pair have been detected in this complex, one of them showing steric hindrance with one of the DMP methoxy groups (Figure 3g). The same dual conformation was detected in the 1-naphthol (complex I) and in the VA complex crystal (Figure $3 \mathrm{e}, \mathrm{h}$ ). This feature is possibly related to the highly dynamic binding indicated above. Finally, the complexes with VA and styrene displayed a similar binding mode, with the primary alcohol group and the vinyl tail located close to the iron and substituting for the heme water, respectively (Figure $3 \mathrm{~h}, \mathrm{i}$ ). In an earlier study, several crystals of cytochrome P450 BM3 treated with styrene presented different modes of binding for this substrate depending on the soaking time. ${ }^{32}$ The position observed in this study was similar to that previously considered as a low-affinity productive binding mode. Consequently, these complexes might be mimicking the corresponding products of the hydroxylation (oxidation of primary alcohols by UPO proceeds via initial hydroxylation to form gem-diols that are in equilibrium with the corresponding free aldehydes) and epoxidation reactions.

\section{DISCUSSION}

Until very recently, peroxygenases were considered as a "hidden treasure trove" within the fungal kingdom. Because of their exquisite $\mathrm{C}-\mathrm{H}$ oxyfunctionalization chemistry, these heme-thiolate enzymes naturally insert oxygen into unactivated aliphatic and aromatic hydrocarbons, fed simply with hydrogen peroxide. As such, the past decade has witnessed intense research into these enzymes, mostly focusing on AaeUPO, the first enzyme of this clade that has been identified. 
a

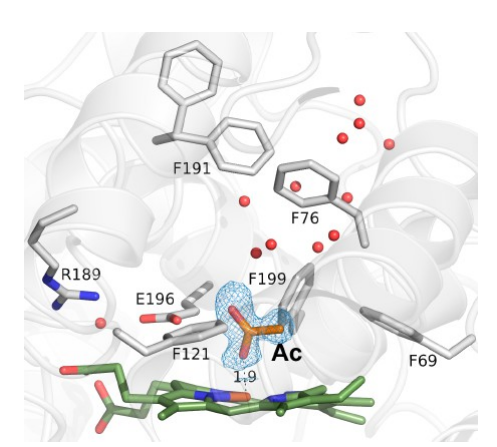

d

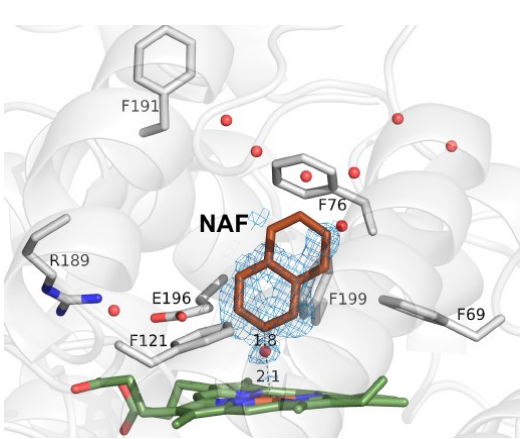

g

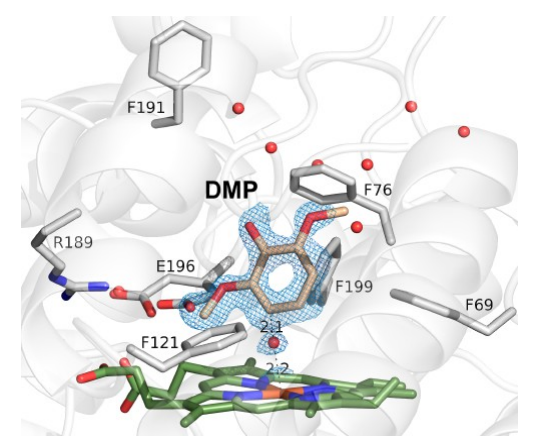

b

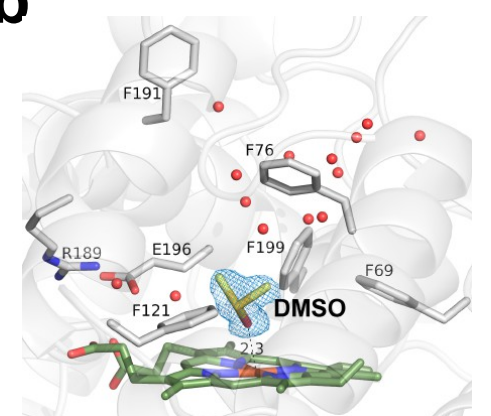

e

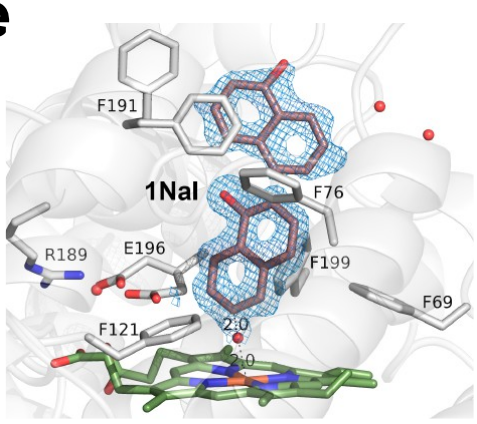

h

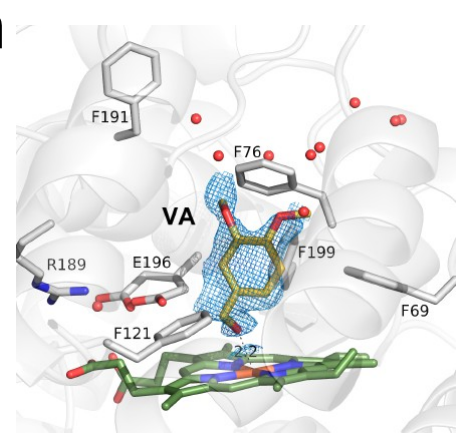

C

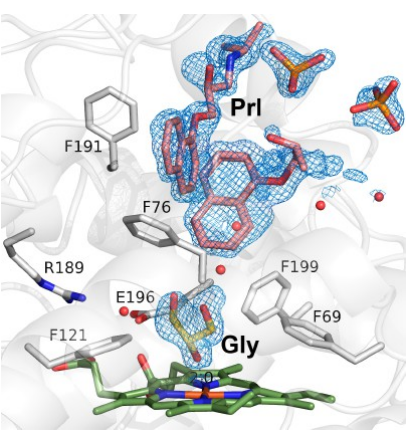

f

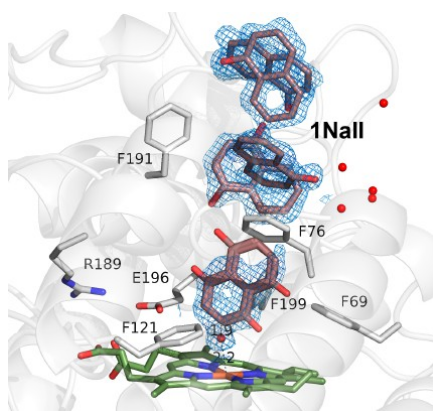

i

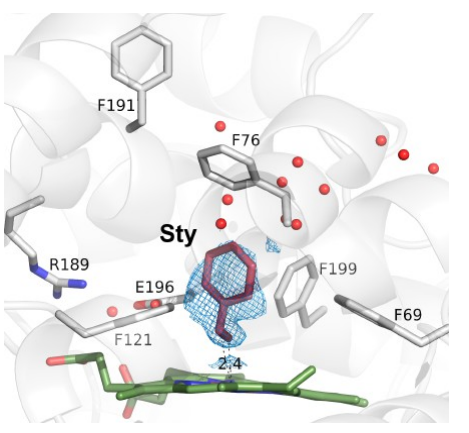

Figure 3. Structural peroxygenase complexes. PaDa-I crystals in complexes with (a) acetate (Ac), (b) DMSO, (c) propranolol (Prl) [with glycerol (Gly)], (d) naphthalene (NAF), (e) 1-naphthol (complex I, 1NaI), (f) 1-naphthol (complex II, 1NaII), (g) DMP, (h) VA, and (i) styrene (Sty). Key residues for binding are represented as sticks, and water molecules as red spheres; the heme group is shown as green sticks. The $2 F_{\mathrm{o}}-F_{\mathrm{c}}$ electronic density at the ligands has been contoured at a root-mean-square deviation of $0.9-1 \sigma$. See also Table 1.

This extensive portfolio of reactions has recently been opened up to industrial needs by evolution of the first fungal laboratory peroxygenase. To aid such developments, it is paramount to understand the pattern of UPO substrate binding to be capable of exploring its substrate and catalytic promiscuity through computational and directed evolution methods. Here, we investigated the substrate binding of UPO by crystallizing, mutating, and complexing our laboratory-evolved peroxygenase with a palette of substrates of different chemical natures.

The PaDa-I variant possesses a unique combination of mutations (V57A-L67F-V75I-I248V-F311L) that improves the functional expression in yeast roughly 1100 -fold, also provoking notable differences in activity. ${ }^{22}$ The distribution of such substitutions in different regions was largely conservative (i.e., equivalent residues in terms of polarity and charge), and they helped to maintain the general structure of the native enzyme. Nevertheless, the presence of this mutational backbone meant that some relevant differences were observed when comparing the crystals of PaDa-I to those of wild type AaeUPO, including a complete $\mathrm{N}$-terminus and a highly dynamic and broader heme access channel.
Although PaDa-I shows rather unspecific and dynamic substrate trafficking, it maintains a common mode of binding to the heme in the different crystal complexes (Figure 4a). Irrespective of the ligands' nature, they were all unequivocally sandwiched between Phe121 and Phe199, also interacting strongly with Phe69. As such, and despite the low specificity of these hydrophobic interactions, the triad of phenylalanines participate in the direct binding of the substrate to the heme. The dual conformation of the catalytic Glu196 observed in some of the complexes (with DMP, 1-naphthol, and VA) may indicate a dynamic catalytic mechanism (with distinct induced fit and plasticity of the UPO protein), although further experimental evidence will be needed to confirm this. Small-chain residues like Ala73 and Thr192 can be found in the neighborhood of the Phe triad, these accommodating the methoxy or hydroxyl groups of some of the ligands through additional hydrophobic or polar contacts.

In terms of the highly dynamic substrate entry and trafficking observed, some of the 1-naphthol and propranolol molecules were trapped within the heme channel in our soaking experiments, and their similar and ordered positions suggest relevant information regarding the diffusion of the 
a

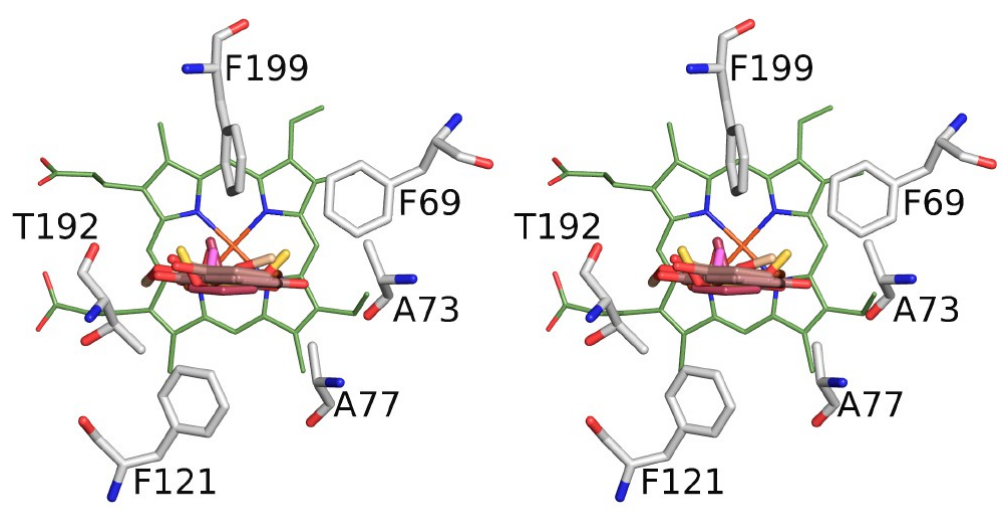

b
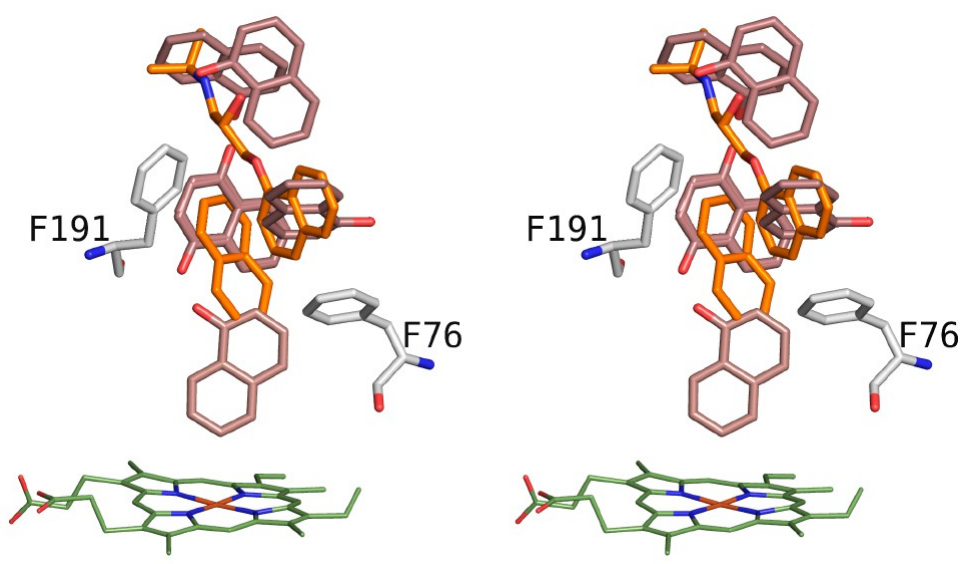

C

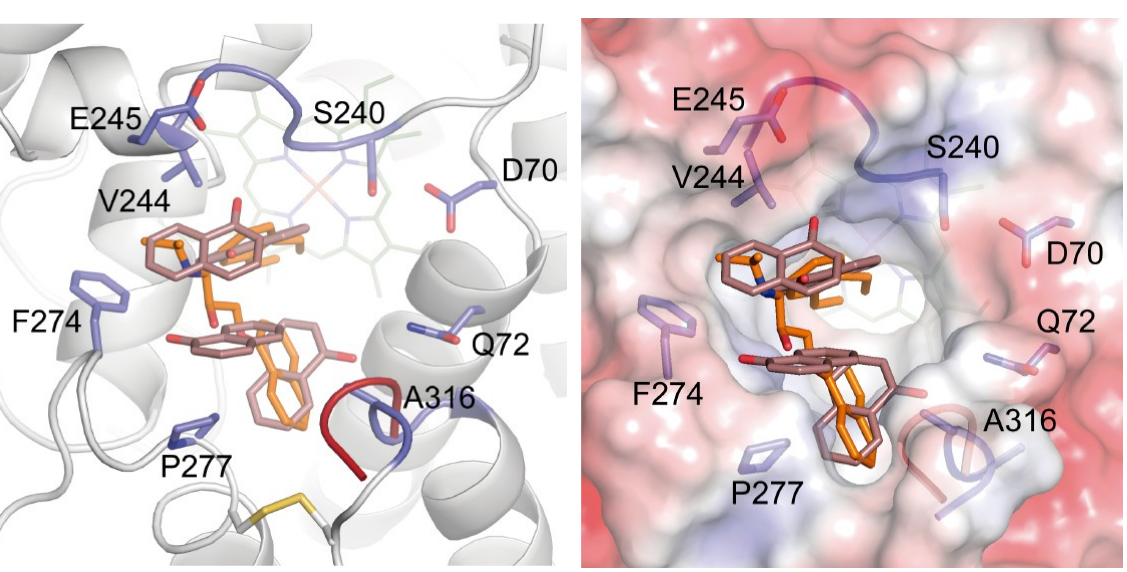

Figure 4. Molecular basis of PaDa-I specificity. (a) Structural superimposition of the different molecules bound at the heme catalytic site reported in this study, represented as a stereopair, showing relevant interacting protein residues. (b) Comparison of bound propranolol and 1-naphthol (complex II) in the corresponding crystal complexes to illustrate the putative transitory sites of binding of ligands to the heme site. (c) Cartoon (left) and surface (right) representation of a $90^{\circ}$ rotation view of panel b, as seen from the outer entrance to the funnel, showing the residues that establish atomic interactions with the ligands at the outer entrance. Residues from loops S240-S245 and G314-G318 contribute to substrate binding by mostly main-chain contacts. The red loop indicates an alternative conformation of loop G314-G318 observed in some complexes, and the yellow sticks indicate the disulfide bridge stabilizing the C-terminus.

substrates toward the heme. The side chains of Phe76 and Phe191 protrude within the channel, and they are involved in strong aromatic interactions that are essential to guide the substrate toward the catalytic site (Figure $4 \mathrm{~b}$ ). Hence, the dual conformational state observed in Phe191 seems to be important in this process. Indeed, in a recent study of a peroxygenase mutant that had evolved to synthesize human drug metabolites from propranolol, we also observed this striking switch of Phe191 in molecular dynamics simulations, which displaced the $\alpha$-helix hosting this residue and, in turn, controlled access of the substrate to the heme. ${ }^{25}$ Conversely, it is reasonable to think that this flexible residue may act as a "molecular hinge", which regulates the trafficking and residence time of substrates and products within the channel, along with Phe76, an effect that seems to be paramount for controlling the enzyme's peroxygenative and peroxidative activity. It is also worth noting that there are a few more hydrophobic residues exposed to the solvent at the outer entrance to the channel (i.e., Val244, Phe274, and Pro277), which precisely position the trapped substrates through direct hydrophobic interactions (Figure 4c). 
As indicated above, and despite its marked hydrophobicity, the channel is filled with water molecules that are displaced by the ligands upon binding. These water molecules are mostly hydrogen bound to main-chain atoms, involving two regions of the peroxygenase in particular: the Ser240-Asp245 and Gly314-Gly318 loops. The former seems to be implicated in the pattern of substrate binding, because the G241D substitution (from a previous directed evolution campaign aimed at naphthalene hydroxylation) induced a shift in the Ser240Asp245 loop, apparently displacing the $\alpha$-helix that harbors the catalytic Glu196-Arg189 acid-base pair. ${ }^{24}$ The latter motif $\left({ }_{314} \mathrm{GVAAG}_{318}\right)$ shapes the heme funnel and is very flexible, with different conformations observed in the crystal complexes (Figure 4c). After the saturation of Ala316 in this loop, a noticeable improvement in both peroxidative and peroxygenative activities was observed in the JEd-I variant, again reflecting the plasticity of this loop (Table 2). In summary, the strongly malleable loops at the outer entrance of the heme channel, along with the dual conformation observed for both the Phe191 side chain and the catalytic Glu196, highlight how dynamic the overall binding is, the main driving force behind the substrate promiscuity of UPO with simultaneous selectivity of these reactions.

Conclusions and Outlook. After more than 10 years of research, we might now consider it inappropriate to refer to fungal peroxygenase as an "unspecific" peroxygenase (UPO), particularly given its strong selectivity for $\mathrm{C}-\mathrm{H}$ oxyfunctionalization (in many cases more enantio- than regioselective). Indeed, this biocatalyst might now be best termed a "promiscuous" peroxygenase. Although only six fungal UPOs have been isolated to date and at least partially characterized, those from $A$. aegerita, ${ }^{14}$ Coprinellus radians, ${ }^{33} \mathrm{M}$. rotula, ${ }^{34}$ Coprinopsis cinerea (recombinant UPO expressed in Aspergillus oryzae), ${ }^{35}$ Chaetomium globosum, ${ }^{36}$ and Marasmius wettsteinii, ${ }^{37}$ new peroxygenases and peroxygenase-like proteins are rapidly emerging from different sources that open the way to engage in "peroxygenase-based" synthetic chemistry. Protein engineering is a valuable technique for adapting peroxygenases to current needs. Accordingly, a multidisciplinary approach that combines directed evolution with structural and computational analysis will help in the development of ad hoc peroxygenases for biotechnology applications. However, the oxidative damage caused by peroxide in peroxygenases on a preparative scale is a serious drawback, and several approaches are being followed to limit this effect. These include the use of enzyme cascade reactions with methanol as the sacrificial electron donor for the reductive activation of $\mathrm{O}_{2}$ or, more recently, the combination of evolved UPOs with inorganic photocatalysts. ${ }^{38-40}$

\section{ASSOCIATED CONTENT}

\section{S Supporting Information}

The Supporting Information is available free of charge on the ACS Publications website at DOI: 10.1021/acschembio.8b00500.

Materials and Methods, Supplementary Figures 1 and 2, and Supplementary Scheme 1 (PDF)

\section{AUTHOR INFORMATION}

\section{Corresponding Authors}

*Institute of Catalysis, CSIC, Marie Curie 2 L10, 28049 Madrid, Spain. E-mail: malcalde@icp.csic.es.

*Institute of Physical Chemistry "Rocasolano", CSIC, Serrano 117, 28006 Madrid, Spain. E-mail: xjulia@iqfr.csic.es.

\section{ORCID}

Miguel Alcalde: 0000-0001-6780-7616

Author Contributions

${ }$ M.R.-E. and P.M.-E. contributed equally to this work.

Notes

The authors declare no competing financial interest.

\section{ACKNOWLEDGMENTS}

This work was supported by the European Union (FP7-KBBE2013-7-613549-INDOX and H2020-BBI-PPP-2015-2-720297ENZOX2), the CSIC (Project PIE-201580E042), and the Spanish Ministry of Economy, Industry and Competitiveness (Projects LIGNOLUTION, BIO2013-48779-C4-2-R, and BIO2016-76601-C3-3-R). The authors thank the Synchrotron Radiation Source at Alba (Barcelona, Spain) for assistance with the BL13-XALOC beamline.

\section{REFERENCES}

(1) Bollinger, J.-M., Jr., and Broderick, J.-B. (2009) Frontiers in enzymatic C-H bond activation. Curr. Opin. Chem. Biol. 13, 51-57.

(2) Roduner, E., Kaim, W., Sarkar, B., Urlacher, V.-B., Pleiss, J., Gläser, R., Einicke, W.-D., Sprenger, G. A., Beifu $\beta$, U., Klemm, E., Liebner, C., Hieronymus, H., Hsu, S.-F., Plietker, B., and Laschat, S. (2013) Selective catalytic oxidation of C-H bonds with molecular oxygen. Chem CatChem 5, 82-112.

(3) Fasan, R. (2012) Tuning P450 enzymes as oxidation catalysts. ACS Catal. 2, 647-666.

(4) Urlacher, V.-B., and Girhard, M. (2012) Cytochrome P450 monooxygenases: an update on perspectives for synthetic applications. Trends Biotechnol. 30, 26-36.

(5) Sakaki, T. (2012) Practical application of cytochrome P450. Biol. Pharm. Bull. 35, 844-849.

(6) Bernhardt, R., and Urlacher, V.-B. (2014) Cytochrome P450 as promising catalysts for biotechnological applications: chances and limitations. Appl. Microbiol. Biotechnol. 98, 6185-6203.

(7) Holtmann, D., and Hollmann, F. (2016) The oxygen dilemma: a severe challenge for the application of monooxygenases? ChemBioChem 17, 1391-1398.

(8) Molina-Espeja, P., Gomez de Santos, P., and Alcalde, M. (2017) Directed evolution of unspecific peroxygenase. In Directed enzyme evolution: Advances and applications (Alcalde, M., Ed.) pp 127-143, Springer International Publishing, Cham, Switzerland.

(9) Hrycay, E.-G., and Bandiera, S.-M. (2012) The monooxygenase, peroxidase, and peroxygenase properties of cytochrome P450. Arch. Biochem. Biophys. 522, 71-89.

(10) Arnold, F.-H. (2018) Directed evolution: Bringing new chemistry to life. Angew. Chem., Int. Ed. 57, 4143-4148.

(11) Xu, H., Ning, L., Yang, W., Fang, B., Wang, C., Wang, Y., Xu, J., Collin, S., Laeuffer, F., Fourage, L., and Li, S. (2017) In vitro oxidative decarboxylation of free fatty acids to terminal alkenes by two new P450 peroxygenases. Biotechnol. Biofuels 10, 208.

(12) Munro, A.-W., McLean, K.-J., Grant, J.-L., and Makris, T.-M. (2018) Structure and function of the cytochrome P450 peroxygenase enzymes. Biochem. Soc. Trans. 46, 183-196.

(13) Onoda, H., Shoji, O., Suzuki, K., Sugimoto, H., Shiro, Y., and Watanabe, Y. (2018) $\alpha$-Oxidative decarboxylation of fatty acids catalyzed by cytochrome $\mathrm{P} 450$ peroxygenases yielding shorter -alkylchain fatty acids. Catal. Sci. Technol. 8, 434-442.

(14) Ullrich, R., Nüske, J., Scheibner, K., Spantzel, J., and Hofrichter, M. (2004) Novel haloperoxidase from the agaric basidiomycete Agrocybe aegerita oxidizes aryl alcohols and aldehydes. Appl. Environ. Microbiol. 70, 4575-4581.

(15) Pecyna, M.-J., Ullrich, R., Bittner, B., Clemens, A., Scheibner, K., Schubert, R., and Hofrichter, M. (2009) Molecular characterization of aromatic peroxygenase from Agrocybe aegerita. Appl. Microbiol. Biotechnol. 84, 885-897. 
(16) Hofrichter, M., Ullrich, R., Pecyna, M.-J., Liers, C., and Lundell, T. (2010) New and classic families of secreted fungal heme peroxidases. Appl. Microbiol. Biotechnol. 87, 871-897.

(17) Hofrichter, M., Kellner, H., Pecyna, M.-J., and Ullrich, R. (2015) Fungal unspecific peroxygenases: Heme-thiolate proteins that combine peroxidase and cytochrome $\mathrm{P} 450$ properties. In Advances in Experimental Medicine and Biology (Hrycay, E.-G., and Bandiera, S.M., Eds.) Vol. 851, pp 341-368, Springer International Publishing, Cham, Switzerland.

(18) Wang, Y., Lan, D., Durrani, R., and Hollmann, F. (2017) Peroxygenases en route to becoming dream catalysts. What are the opportunities and challenges? Curr. Opin. Chem. Biol. 37, 1-9.

(19) Hofrichter, M., and Ullrich, R. (2014) Oxidations catalyzed by fungal peroxygenases. Curr. Opin. Chem. Biol. 19, 116-125.

(20) Bormann, S., Gomez Baraibar, A., Ni, Y., Holtmann, D., and Hollmann, F. (2015) Specific oxyfunctionalisations catalysed by peroxygenases: opportunities, challenges and solutions. Catal. Sci. Technol. 5, 2038-2052.

(21) Ullrich, R., Poraj-Kobielska, M., Scholze, S., Halbout, C., Sandvoss, M., Pecyna, M.-J., Scheibner, K., and Hofrichter, M. (2018) Side chain removal from corticosteroids by unspecific peroxygenase. J. Inorg. Biochem. 183, 84-93.

(22) Molina-Espeja, P., Garcia-Ruiz, E., Gonzalez-Perez, D., Ullrich, R., Hofrichter, M., and Alcalde, M. (2014) Directed evolution of unspecific peroxygenase from Agrocybe aegerita. Appl. Environ. Microbiol. 80, 3496-3507.

(23) Molina-Espeja, P., Ma, S., Mate, D.-M., Ludwig, R., and Alcalde, M. (2015) Tandem-yeast expression system for engineering and producing unspecific peroxygenase. Enzyme Microb. Technol. 73-74, $29-33$.

(24) Molina-Espeja, P., Cañellas, M., Plou, F.-J., Hofrichter, M., Lucas, F., Guallar, V., and Alcalde, M. (2016) Synthesis of 1-naphthol by a natural peroxygenase engineered by directed evolution. ChemBioChem 17, 341-349.

(25) Gomez de Santos, P., Cañellas, M., Tieves, F., Younes, S.-H.-H., Molina-Espeja, P., Hofrichter, M., Hollmann, F., Guallar, V., and Alcalde, M. (2018) Selective synthesis of the human drug metabolite 5 '-hydroxypropranolol by and evolved self-sufficient peroxygenase. ACS Catal. 8, 4789-4799.

(26) Piontek, K., Strittmatter, E., Ullrich, R., Gröbe, G., Pecyna, M.J., Kluge, M., Scheibner, K., Hofrichter, M., and Plattner, D.-A. (2013) Structural basis of substrate conversion in a new aromatic peroxygenase cytochrome $\mathrm{P} 450$ functionality with benefits. J. Biol. Chem. 288, 34767-34776.

(27) Molina-Espeja, P., Plou, F. J., Gomez de Santos, P., and Alcalde, M. (2017) Mutants of unspecific peroxygenase with high monooxygenase activity and uses thereof. Patent WO/2017/081355.

(28) Andreeva, A., Howorth, D., Chothia, C., Kulesha, E., and Murzin, A.-G. (2014) SCOP2 prototype: a new approach to protein structure mining. Nucleic Acids Res. 42, D310-D314.

(29) Kühnel, K., Blankenfeldt, W., Terner, J., and Schlichting, I. (2006) Crystal Structures of Chloroperoxidase with Its Bound Substrates and Complexed with Formate, Acetate, and Nitrate. J. Biol. Chem. 281, 23990-23998.

(30) Kühnel, K., Derat, E., Terner, J., Shaik, S., and Schlichting, I. (2007) Structure and quantum chemical characterization of chloroperoxidase compound 0 , a common reaction intermediate of diverse heme enzymes. Proc. Natl. Acad. Sci. U. S. A. 104, 99-104.

(31) Yoshida, T., Tsuge, H., Hisabori, T., and Sugano, Y. (2012) Crystal structures of dye-decolorizing peroxidase with ascorbic acid and 2,6-dimethoxyphenol. FEBS Lett. 586, 4351-4356.

(32) Shehzad, A., Panneerselvam, S., Linow, M., Bocola, M., Roccatano, D., Mueller-Dieckmann, J., Wilmanns, M., and Schwaneberg, U. (2013) P450 BM3 crystal structures reveal the role of the charged surface residue Lys/Arg184 in inversion of enantioselective styrene epoxidation. Chem. Commun. 49, 4694-4696. (33) Anh, D.-H., Ullrich, R., Benndorf, D., Svatós, A., Muck, A., and Hofrichter, M. (2007) The coprophilous mushroom Coprinus radians secretes a haloperoxidase that catalyzes aromatic peroxygenation. Appl. Environ. Microbiol. 73, 5477-5485.

(34) Gröbe, G., Ullrich, R., Pecyna, M.-J., Kapturska, D., Friedrich, S., Hofrichter, M., and Scheibner, K. (2011) High-yield production of aromatic peroxygenase by the agaric fungus Marasmius rotula. $A M B$ Express 1, 31.

(35) Babot, E.-D., del Rio, J.-C., Kalum, L., Martinez, A.-T., and Gutierrez, A. (2013) Oxyfunctionalization of aliphatic compounds by a recombinant peroxygenase from Coprinopsis cinerea. Biotechnol. Bioeng. 110, 2323-2332.

(36) Kiebist, J., Schmidtke, K.-U., Zimmermann, J., Kellner, H., Jehmlich, N., Ullrich, R., Zänder, D., Hofrichter, M., and Scheibner, K. (2017) A peroxygenase from Chaetomium globosum catalyzes the selective oxygenation of testosterone. ChemBioChem 18, 563-569.

(37) Ullrich, R., Poraj-Kobielska, M., Scholze, S., Halbout, C., Sandvoss, M., Pecyna, M.-J., Scheibner, K., and Hofrichter, M. (2018) Side chain removal from corticosteroids by unspecific peroxygenase. J. Inorg. Biochem. 183, 84-93.

(38) Ni, Y., Fernández-Fueyo, E., Gomez Baraibar, A., Ullrich, R. Hofrichter, M., Yanase, H., Alcalde, M., van Berkel, W.-J.-H., and Hollmann, F. (2016) Peroxygenase-catalyzed oxyfunctionalization reactions promoted by the complete oxidation of methanol. Angew. Chem., Int. Ed. 55, 798-801.

(39) Zhang, W., Burek, B.-O., Fernandez-Fueyo, E., Alcalde, M., Bloh, J.-Z., and Hollmann, F. (2017) Selective activation of C-H bonds by cascading photochemistry with biocatalysis. Angew. Chem., Int. Ed. 56, 15451-15455.

(40) Zhang, W., Fernandez-Fueyo, E., Ni, Y., van Schie, M., Gacs, J., Renirie, R., Wever, R., Mutti, F.-G., Rother, D., Alcalde, M., and Hollmann, F. (2018) Selective aerobic oxidation reactions using a combination of photocatalytic water oxidation and enzymatic oxyfunctionalizations. Nat. Catal. 1, 55-62. 